\title{
Numeration systems and fractal sequences
}

by

\author{
Clark Kimberling (Evansville, Ind.)
}

Let $\mathbb{N}$ denote the set of positive integers. Every sequence $\mathbb{B}=\left(b_{0}, b_{1}, \ldots\right)$ of numbers in $\mathbb{N}$ satisfying

$$
1=b_{0}<b_{1}<\ldots
$$

is a basis for $\mathbb{N}$, as each $n$ in $\mathbb{N}$ has a $\mathbb{B}$-representation

$$
n=c_{0} b_{0}+c_{1} b_{1}+\ldots+c_{k} b_{k},
$$

where $b_{k} \leq n<b_{k+1}$ and the coefficients $c_{i}$ are given by the division algorithm:

$$
n=c_{k} b_{k}+r_{k}, \quad c_{k}=\left[n / b_{k}\right], \quad 0 \leq r_{k}<b_{k}
$$

and

$$
r_{i}=c_{i-1} b_{i-1}+r_{i-1}, \quad c_{i-1}=\left[r_{i} / b_{i-1}\right], \quad 0 \leq r_{i-1}<b_{i-1}
$$

for $1 \leq i<k$. In (2) let $i$ be the least index $h$ such that $c_{h} \neq 0$; then $b_{i}$ is the $\mathbb{B}$-residue of $n$. A proper basis is a basis other than the sequence $(1,2, \ldots)$ consisting of all the positive integers.

We extend the above notions to finite sequences $\mathbb{B}_{j}=\left(b_{0}, b_{1}, \ldots, b_{j}\right)$ satisfying

$$
1=b_{0}<b_{1}<\ldots<b_{j}
$$

for $j \geq 0$. Such a finite sequence is a finite basis, and a $\mathbb{B}_{j}$-representation is a sum

$$
c_{0} b_{0}+c_{1} b_{1}+\ldots+c_{j} b_{j}
$$

such that if $n=c_{0} b_{0}+c_{1} b_{1}+\ldots+c_{j} b_{j}$, then there exist integers $r_{0}, r_{1}, \ldots, r_{j}$ such that

$$
n=c_{j} b_{j}+r_{j}, \quad c_{j}=\left[n / b_{j-1}\right], \quad 0 \leq r_{j}<b_{j}
$$

1991 Mathematics Subject Classification: Primary 11B75; Secondary 11B37.

Key words and phrases: fractal sequence, fractal basis, interspersion, linear recurrence, numeration system. 
and

$$
r_{i}=c_{i-1} b_{i-1}+r_{i-1}, \quad c_{i-1}=\left[r_{i} / b_{i-1}\right], \quad 0 \leq r_{i-1}<b_{i-1}
$$

for $1 \leq i \leq j$.

From any basis or finite basis $\mathbb{B}$ we construct an array $A(\mathbb{B})$ of numbers $a(i, j)$ here called the $\mathbb{B}$-numeration system. Row 1 of $A(\mathbb{B})$ is the basis $\mathbb{B}$; i.e., $a(1, j)=b_{j-1}$, for $j=1,2, \ldots$ Column 1 is the ordered residue class containing 1 ; i.e., $a(i, 1)$ is the $i$ th number $n$ whose $\mathbb{B}$-residue is 1 . Generally, column $j$ is the ordered residue class whose least element is $b_{j-1}$, so that $a(i, j)$ is the $i$ th number $n$ whose $\mathbb{B}$-residue is $b_{j-1}$. Note that every $n$ in $\mathbb{N}$ occurs exactly once in $A(\mathbb{B})$. As an example, the first six rows of the $\mathbb{B}$-numeration system of the finite basis $\mathbb{B}=(1,2,3,5,8,13)$ are

$\begin{array}{rrrrrr}1 & 2 & 3 & 5 & 8 & 13 \\ 4 & 7 & 11 & 18 & 21 & 26 \\ 6 & 10 & 16 & 31 & 34 & 39 \\ 9 & 15 & 24 & 44 & 47 & 52 \\ 12 & 20 & 29 & 57 & 60 & 65 \\ 14 & 23 & 37 & 70 & 73 & 78\end{array}$

A $\mathbb{B}$-numeration system can also be represented as a sequence $S(\mathbb{B})=$ $\left(s_{1}, s_{2}, \ldots\right)$, where

$s_{n}$ is the number of the row of the array $A(\mathbb{B})$ in which $n$ occurs; i.e., if $n=a(i, j)$, then $s_{n}=i$. We call $S(\mathbb{B})$ the paraphrase of $\mathbb{B}$. For example, the paraphrase of the finite basis $(1,2,3,5,8,13)$ begins with

$$
111213214325164372852 \text {. }
$$

As a second example, let $\mathbb{B}$ be the basis for the ordinary binary system:

$$
\mathbb{B}=\left(1,2,2^{2}, 2^{3}, 2^{4}, 2^{5}, \ldots\right) ;
$$

in this case, $S(\mathbb{B})$ begins with

(6) 112132415362748195103116122137144158161 .

Now suppose $S=\left(s_{1}, s_{2}, \ldots\right)$ is any sequence such that for every $i$ in $\mathbb{N}$ there are infinitely many $n$ such that $s_{n}=i$; and further, that if $i+1=s_{n}$, then $i=s_{m}$ for some $m<n$. The upper-trimmed subsequence of $S$ is the sequence $\Lambda(S)$ obtained from $S$ by deleting the first occurrence of $n$, for each $n$. If $\Lambda(S)=S$, then $S$ is a fractal sequence, so named, in [3], because the self-similarity property $\Lambda(S)=S$ implies that $S$ contains a copy of itself, and hence contains infinitely many copies of itself. The sequence begun in (6), and also the paraphrases of trinary and the other -ary number systems, are examples of fractal sequences. Another familiar sequence that is a fractal basis is the sequence $(1,2,3,5,8,13,21, \ldots)$ of Fibonacci numbers. 
To determine which bases are fractal bases, we shall extend finite bases one term at a time, with attention to certain shift functions. To define them, let $\mathbb{B}_{j}=\left(b_{0}, b_{1}, \ldots, b_{j}\right)$, where $j \geq 1$, and for each $n$ in $\mathbb{N}$, let the $\mathbb{B}_{j-1}$-representation of $n$ be given by

$$
n=\sum_{h=0}^{j-1} c_{h} b_{h}
$$

then the shift-function $f_{\mathbb{B}_{j}}$ is defined by

$$
f_{\mathbb{B}_{j}}(n)=\sum_{h=0}^{j-1} c_{h} b_{h+1} .
$$

We call $\mathbb{B}_{j}$ an affable finite basis if the sum in (8) is a $\mathbb{B}_{j}$-representation whenever the sum in $(7)$ is a $\mathbb{B}_{j-1}$-representation. To see what can go wrong, consider the finite basis $\mathbb{B}_{j}=\mathbb{B}_{3}=(1,3,6,10)$ : here the $\mathbb{B}_{2}$-representation of 5 is $2 \cdot 1+1 \cdot 3$, so that $f_{\mathbb{B}_{2}}(5)=2 \cdot 3+1 \cdot 6=12$; but alas, the $\mathbb{B}_{3}$-representation of 12 is $2 \cdot 1+1 \cdot 10$, not $2 \cdot 3+1 \cdot 6$. Theorem 1 gives lower bounds on successive $b_{i}$ 's that ensure that $\mathbb{B}_{j}$ is affable.

LEMMA 1. If the sum in (2) is a $\mathbb{B}$-representation, then

$$
\sum_{h=0}^{i} c_{h} b_{h}<b_{i+1}
$$

for $i=0,1, \ldots, k$; conversely, if (9) holds for $i=0,1, \ldots, k$, then each of the $k+1$ sums is a $\mathbb{B}$-representation. Similarly, if $n<b_{j}$ and the sum in $\left(2^{\prime}\right)$ is a $\mathbb{B}_{j}$-representation, then (9) holds for $i=0,1, \ldots, j-1$; and conversely, if (9) holds for $i=0,1, \ldots, j-1$, then each of the $j$ sums is a $\mathbb{B}_{j}$-representation.

Pr o of. The proof for $\mathbb{B}$-representations is essentially given in [4]. A similar proof for $\mathbb{B}_{j}$-representations is given here for the sake of completeness. First, suppose $n<b_{j}$ and that $n$ equals the sum $\left(2^{\prime}\right)$, a $\mathbb{B}_{j}$-representation. Then by $\left(4^{\prime}\right)$,

$$
\begin{aligned}
b_{1} & >r_{1}=c_{0} b_{0}, \\
b_{2}> & r_{2}=c_{1} b_{1}+r_{1}=c_{1} b_{1}+c_{0} b_{0}, \\
b_{3}> & r_{3}=c_{2} b_{2}+r_{2}=c_{2} b_{2}+c_{1} b_{1}+c_{0} b_{0}, \\
& \vdots \\
b_{j-1} & >r_{j-1}=c_{j-2} b_{j-2}+r_{j-2}=\sum_{h=0}^{j-2} c_{h} b_{h} .
\end{aligned}
$$

These $j-1$ inequalities together with $n<b_{j}$ show that (9) holds for $i=$ $0,1, \ldots, j-1$. 
For the converse, suppose $c_{0}, c_{1}, \ldots, c_{j-1}$ are nonnegative integers such that the sum in $(9)$ is a $\mathbb{B}_{j}$-representation for $i=0,1, \ldots, j-1$. Let $r_{0}=0$ and

$$
r_{i}=c_{0} b_{0}+c_{1} b_{1}+\ldots+c_{i-1} b_{i-1}
$$

for $1 \leq i<j$. Clearly $r_{0}<b_{0}$, and $r_{i-1}<b_{i-1}$ for $i=2,3, \ldots, j$, by (9), so that conditions $\left(4^{\prime}\right)$ hold. Write the sum $c_{0} b_{0}+c_{1} b_{1}+\ldots+c_{j-1} b_{j-1}$ as $n$; then condition $\left(3^{\prime}\right)$ holds, since $r_{j}<b_{j}$, by $(9)$.

Theorem 1. Suppose $j \geq 2$. Let $\mathbb{B}_{j}=\left(b_{0}, b_{1}, \ldots, b_{j}\right)$ be a finite basis. The following statements are equivalent:

(i) $\mathbb{B}_{j}$ is an affable finite basis.

(ii) If $c_{0}, c_{1}, \ldots, c_{j-2}$ are nonnegative integers satisfying the $j-1$ inequalities

$$
\begin{gathered}
c_{0} b_{0}<b_{1}, \\
c_{0} b_{0}+c_{1} b_{1}<b_{2}, \\
\vdots \\
c_{0} b_{0}+c_{1} b_{1}+\ldots+c_{j-2} b_{j-2}<b_{j-1},
\end{gathered}
$$

then the following $j-1$ inequalities also hold:

$$
\begin{gathered}
c_{0} b_{1}<b_{2}, \\
c_{0} b_{1}+c_{1} b_{2}<b_{3}, \\
\vdots \\
c_{0} b_{1}+c_{1} b_{2}+\ldots+c_{j-2} b_{j-1}<b_{j} .
\end{gathered}
$$

(iii) $b_{i} \geq f_{\mathbb{B}_{i-1}}\left(b_{i-1}-1\right)+1$ for $i=2, \ldots, j$.

(iv) $f_{\mathbb{B}_{j}}$ is strictly increasing on the set $\left\{m \in \mathbb{N}: 1 \leq m \leq b_{j-1}\right\}$.

Proof. A proof is given in four parts: (i) $\Leftrightarrow($ ii), (iii) $\Rightarrow$ (ii) and (iv), (i) $\Rightarrow$ (iii), and (iv) $\Rightarrow$ (iii).

P art 1: (i) $\Leftrightarrow\left(\right.$ ii). Suppose $\mathbb{B}_{j}$ is an affable finite basis and $c_{0}, c_{1}, \ldots, c_{j-2}$ are nonnegative integers satisfying $\sum_{h=0}^{i} c_{h} b_{h}<b_{i+1}$ for $i=0,1, \ldots, j-2$. By Lemma 1 , each of these $j-1$ sums is a $\mathbb{B}_{j-1}$-representation. Since $\mathbb{B}_{j}$ is affable, each of the sums $\sum_{h=0}^{i} c_{h} b_{h+1}$, for $i=0,1, \ldots, j-2$, is a $\mathbb{B}_{j}$-representation, so that by Lemma 1 ,

$$
\begin{gathered}
c_{0} b_{1}<b_{2}, \\
c_{0} b_{1}+c_{1} b_{2}<b_{3}, \\
\vdots \\
c_{0} b_{1}+c_{1} b_{2}+\ldots+c_{j-2} b_{j-1}<b_{j},
\end{gathered}
$$

and (ii) holds. 
Now suppose $n$ is given by a $\mathbb{B}_{j-1}$-representation as in (7). Then the $j-1$ inequalities in the hypothesis of (ii) hold, by definition of $\mathbb{B}_{j-1^{-}}$ representation. So, the $j-1$ inequalities in the conclusion of (ii) hold. These are precisely the inequalities that must be satisfied for the sum in (8) to be a $\mathbb{B}_{j}$-representation.

P art 2: (iii) $\Rightarrow$ (ii) and (iv). Suppose $\mathbb{B}_{j}$ is a finite basis and $c_{0}, c_{1}, \ldots, c_{j-2}$ are nonnegative integers satisfying $\sum_{h=0}^{i} c_{h} b_{h}<b_{i+1}$ for $i=0,1, \ldots, j-$ 1. As a first step in an induction argument, assume that $b_{0} c_{0}<b_{1}$. By definition of basis, $b_{0}=1$ and $b_{1} \geq 2$, and by hypothesis,

$$
b_{2} \geq f_{\mathbb{B}_{1}}\left(b_{1}-1\right)+1= \begin{cases}3 & \text { if } b_{1}=2, \\ b_{1}^{2}-b_{1}+1 & \text { if } b_{1} \geq 3 .\end{cases}
$$

If $b_{1}=2$, then $c_{0} b_{0}<b_{1}$ implies $c_{0}=1$, so that $c_{0} b_{1}<b_{1}+1 \leq b_{2}$, as desired. Otherwise, $b_{1} \geq 3$, so that $c_{0} \leq b_{1}-1$, and $c_{0} b_{1} \leq b_{1}^{2}-b_{1}<b_{2}$, as desired. As a first step toward proving (iv), if $c_{0} b_{0}<c_{0}^{\prime} b_{0}$, then clearly $c_{0} b_{1}<c_{0}^{\prime} b_{1}$.

We shall now use a bipartite induction hypothesis.

Hypothesis I. If $h \leq j-1$ and the $h-2$ inequalities

$$
\begin{gathered}
c_{0} b_{0}<b_{1}, \\
c_{0} b_{0}+c_{1} b_{1}<b_{2}, \\
\vdots \\
c_{0} b_{0}+c_{1} b_{1}+\ldots+c_{h-3} b_{h-3}<b_{h-2}
\end{gathered}
$$

hold, then also the following $h-2$ inequalities hold:

$$
\begin{gathered}
c_{0} b_{1}<b_{2}, \\
c_{0} b_{1}+c_{1} b_{2}<b_{3}, \\
\vdots \\
c_{0} b_{1}+c_{1} b_{2}+\ldots+c_{h-3} b_{h-2}<b_{h-1} .
\end{gathered}
$$

Hypothesis II. If $c_{0}^{\prime}, c_{1}^{\prime}, \ldots, c_{h-3}^{\prime}$ are nonnegative integers such that $c_{0}^{\prime} b_{0}+c_{1}^{\prime} b_{1}+\ldots+c_{h-3}^{\prime} b_{h-3}$ is a $\mathbb{B}_{h-3}$-representation, and the $h-2$ inequalities

$$
\begin{aligned}
c_{0} b_{0} & <c_{0}^{\prime} b_{0}, \\
c_{0} b_{0}+c_{1} b_{1} & <c_{0}^{\prime} b_{0}+c_{1}^{\prime} b_{1}, \\
& \vdots \\
c_{0} b_{0}+c_{1} b_{1}+\ldots+c_{h-3} b_{h-3} & <c_{0}^{\prime} b_{0}+c_{1}^{\prime} b_{1}+\ldots+c_{h-3}^{\prime} b_{h-3}
\end{aligned}
$$


hold, then also the following $h-2$ inequalities hold:

$$
\begin{aligned}
c_{0} b_{1} & <c_{0}^{\prime} b_{1}, \\
c_{0} b_{1}+c_{1} b_{2} & <c_{0}^{\prime} b_{1}+c_{1}^{\prime} b_{2}, \\
& \vdots \\
c_{0} b_{1}+c_{1} b_{2}+\ldots+c_{h-3} b_{h-2} & <c_{0}^{\prime} b_{1}+c_{1}^{\prime} b_{2}+\ldots+c_{h-3}^{\prime} b_{h-2} .
\end{aligned}
$$

Now suppose that the $h-1$ inequalities

$$
\begin{gathered}
c_{0} b_{0}<b_{1}, \\
c_{0} b_{0}+c_{1} b_{1}<b_{2}, \\
\vdots \\
c_{0} b_{0}+c_{1} b_{1}+\ldots+c_{h-3} b_{h-3}<b_{h-2}, \\
c_{0} b_{0}+c_{1} b_{1}+\ldots+c_{h-3} b_{h-3}+c_{h-2} b_{h-2}<b_{h-1}
\end{gathered}
$$

have been shown to hold. There are $h-1$ inequalities to be proved. The first $h-2$ hold by Hypothesis I, and we now wish to see that the remaining inequality holds, namely

$$
c_{0} b_{1}+c_{1} b_{2}+\ldots+c_{h-2} b_{h-1}<b_{h} .
$$

Let $d_{0} b_{0}+d_{1} b_{1}+\ldots+d_{h-2} b_{h-2}$ be the $\mathbb{B}_{h-2}$-representation of $b_{h-1}-1$. Then

$$
c_{0} b_{0}+c_{1} b_{1}+\ldots+c_{h-2} b_{h-2} \leq d_{0} b_{0}+d_{1} b_{1}+\ldots+d_{h-2} b_{h-2} .
$$

Case 1: $d_{h-2}=c_{h-2}$. In this case, (11) implies

$$
c_{0} b_{0}+c_{1} b_{1}+\ldots+c_{h-3} b_{h-3} \leq d_{0} b_{0}+d_{1} b_{1}+\ldots+d_{h-3} b_{h-3},
$$

which by Hypothesis II yields

$$
c_{0} b_{1}+c_{1} b_{2}+\ldots+c_{h-3} b_{h-2} \leq d_{0} b_{1}+d_{1} b_{2}+\ldots+d_{h-3} b_{h-2} .
$$

We add $c_{h-2} b_{h-1}=d_{h-2} b_{h-1}$ to both sides to obtain

$$
\begin{aligned}
c_{0} b_{1}+c_{1} b_{2}+\ldots+c_{h-2} b_{h-1} & \leq d_{0} b_{1}+d_{1} b_{2}+\ldots+d_{h-2} b_{h-1} \\
& =f_{\mathbb{B}_{h-1}}\left(b_{h-1}-1\right)<b_{h},
\end{aligned}
$$

so that (10) holds.

Case 2: $\quad d_{h-2}>c_{h-2}$. Since $c_{0} b_{0}+c_{1} b_{1}+\ldots+c_{h-3} b_{h-3}<b_{h-2}$, we have $c_{0} b_{1}+c_{1} b_{2}+\ldots+c_{h-3} b_{h-2}<b_{h-1}$, by Hypothesis I. Then

$$
\begin{aligned}
c_{0} b_{1}+ & c_{1} b_{2}+\ldots+c_{h-3} b_{h-2} \\
& \leq\left(d_{h-2}-c_{h-2}\right) b_{h-1} \\
& \leq d_{0} b_{1}+d_{1} b_{2}+\ldots+d_{h-3} b_{h-2}+\left(d_{h-2}-c_{h-2}\right) b_{h-1},
\end{aligned}
$$

from which (10) follows as at the end of Case 1. 
Case 3: $d_{h-2}<c_{h-2}$. We rewrite (11) as $c_{0} b_{0}+c_{1} b_{1}+\ldots+c_{h-3} b_{h-3}+\left(c_{h-2}-d_{h-2}\right) b_{h-2}<d_{0} b_{0}+d_{1} b_{1}+\ldots+d_{h-3} b_{h-3}$.

This implies $b_{h-2}<d_{0} b_{0}+d_{1} b_{1}+\ldots+d_{h-3} b_{h-3}$, but this violates the premise that the sum $d_{0} b_{0}+d_{1} b_{1}+\ldots+d_{h-2} b_{h-2}$, and therefore also $d_{0} b_{0}+$ $d_{1} b_{1}+\ldots+d_{h-3} b_{h-3}$, is a $\mathbb{B}_{h-2}$-representation. Therefore Case 3 does not occur.

A proof of (ii) is now finished, and we continue with a proof of (iv). Suppose $c_{0}^{\prime}, c_{1}^{\prime}, \ldots, c_{j-2}^{\prime}$ are nonnegative integers and

$$
c_{0} b_{0}+c_{1} b_{1}+\ldots+c_{j-2} b_{j-2}<c_{0}^{\prime} b_{0}+c_{1}^{\prime} b_{1}+\ldots+c_{j-2}^{\prime} b_{j-2}<b_{j-1},
$$

where both sums are $\mathbb{B}_{j-1}$-representations.

Case 1.1: $c_{j-2}^{\prime}=c_{j-2}$. Here $c_{0} b_{0}+c_{1} b_{1}+\ldots+c_{j-3} b_{j-3}<c_{0}^{\prime} b_{0}+c_{1}^{\prime} b_{1}+$ $\ldots+c_{j-3}^{\prime} b_{j-3}$, which by Hypothesis II yields

$$
c_{0} b_{1}+c_{1} b_{2}+\ldots+c_{j-3} b_{j-2}<c_{0}^{\prime} b_{1}+c_{1}^{\prime} b_{2}+\ldots+c_{j-3}^{\prime} b_{j-2} .
$$

We add $c_{j-2} b_{j-1}=c_{j-2}^{\prime} b_{j-1}$ to both sides to obtain

$$
c_{0} b_{1}+c_{1} b_{2}+\ldots+c_{j-2} b_{j-1}<c_{0}^{\prime} b_{1}+c_{1}^{\prime} b_{2}+\ldots+c_{j-2}^{\prime} b_{j-1} .
$$

This proof of (12) for Case 1.1 is obviously very similar to that for Case 1 above. Cases 2.1 and 3.1 are similar to the previous Cases 2 and 3 , and corresponding proofs of (12) are omitted. Now suppose $1 \leq m<n \leq b_{j-1}$. Write $\mathbb{B}_{j}$-representations for $m$ and $n$ :

$$
\begin{aligned}
& m=c_{0} b_{0}+\ldots+c_{j-2} b_{j-2}, \\
& n= \begin{cases}c_{0}^{\prime} b_{0}+\ldots+c_{j-2}^{\prime} b_{j-2} & \text { if } n<b_{j-1}, \\
b_{j-1} & \text { otherwise }\end{cases}
\end{aligned}
$$

and let

$$
\begin{aligned}
& h=\max \left\{i: c_{i} \neq 0, i \leq j-2\right\}, \\
& k= \begin{cases}\max \left\{i: c_{i}^{\prime} \neq 0, i \leq j-2\right\} & \text { if } n<b_{j-1}, \\
j-1 & \text { otherwise. }\end{cases}
\end{aligned}
$$

Case 1.2: $h=k$. In this case, $f_{\mathbb{B}_{j}}(m)<f_{\mathbb{B}_{j}}(n)$ by $(12)$.

Case 2.2: $h<k$ (the case $h>k$ is similar and omitted). Here, $m \leq$ $b_{k}<n$ or $m<b_{k} \leq n$, so that $f_{\mathbb{B}_{j}}(m) \leq f_{\mathbb{B}_{j}}\left(b_{k}\right)=b_{k+1} \leq f_{\mathbb{B}_{j}}(n)$, with strict inequality in at least one place, and a proof of (iv) is finished.

Part 3: (i) $\Rightarrow$ (iii). Suppose $2 \leq i \leq j$. Then $b_{i-1}-1$ has a $\mathbb{B}_{i-2^{-}}$ representation $c_{0} b_{0}+c_{1} b_{1}+\ldots+c_{i-2} b_{i-2}$. By Lemma 1 (reading $i-1$ for $j$ ), we have $i-1$ inequalities: 


$$
\begin{gathered}
c_{0} b_{0}<b_{1}, \\
c_{0} b_{0}+c_{1} b_{1}<b_{2}, \\
\vdots \\
c_{0} b_{0}+c_{1} b_{1}+\ldots+c_{i-2} b_{i-2}<b_{i-1} .
\end{gathered}
$$

By (i), the representation for $f_{\mathbb{B}_{i-1}}\left(b_{i-1}-1\right)$ as $c_{0} b_{1}+c_{1} b_{2}+\ldots+c_{i-2} b_{i-1}$ is a $\mathbb{B}_{i-1}$-representation, and by (ii), already proved to follow from (i), we have $f_{\mathbb{B}_{i-1}}\left(b_{i-1}-1\right)<b_{i}$, and (iii) holds.

P art 4: (iv) $\Rightarrow\left(\right.$ iii). For $i=2,3, \ldots, j$, if (iv) holds then $f_{\mathbb{B}_{i-1}}\left(b_{i-1}-\right.$ $1)=f_{\mathbb{B}_{j}}\left(b_{i-1}-1\right)<f_{\mathbb{B}_{j}}\left(b_{i-1}\right)=b_{i}$, so that (iii) holds.

DEFinitions. We extend the notion of affability given earlier: an infinite basis $\mathbb{B}=\left(b_{0}, b_{1}, \ldots\right)$ is an affable basis if the sum $\sum_{h=0}^{k} c_{h} b_{h+1}$ is a $\mathbb{B}$ representation whenever the sum $\sum_{h=0}^{k} c_{h} b_{h}$ is a $\mathbb{B}$-representation. The notion of shift-function is extended also:

if $n=\sum_{h=0}^{k} c_{h} b_{h}$ is a $\mathbb{B}$-representation, then $f_{\mathbb{B}}(n)=\sum_{h=0}^{k} c_{h} b_{h+1}$.

Theorem 2. Let $\mathbb{B}=\left(b_{0}, b_{1}, \ldots\right)$ be a basis, and let $\mathbb{B}_{j}=\left(b_{0}, b_{1}, \ldots, b_{j}\right)$ for $j \geq 2$. The following statements are equivalent:

(i) $\mathbb{B}$ is an affable basis.

(ii) $\mathbb{B}_{j}$ is an affable finite basis for all $j \geq 2$.

(iii) $b_{j} \geq f_{\mathbb{B}_{j-1}}\left(b_{j-1}-1\right)+1$ for all $j \geq 2$.

(iv) $f_{\mathbb{B}}$ is strictly increasing on $\mathbb{N}$.

Proof. This follows easily from Theorem 1.

Definitions. Suppose $S=\left(s_{n}\right)$ is a sequence (possibly finite) of numbers in $\mathbb{N}$. The counting array of $S$ is the array $C(S)$ with terms $a(i, j)$ given by

$a(i, j)$ is the index $n$ for which $s_{n}$ is the $j$ th occurrence of $i$ in $S$.

Note that if $S$ is the paraphrase of an infinite basis $\mathbb{B}$, then

$$
C(S)=A(\mathbb{B}) \quad \text { and } \quad \mathbb{B}=(a(1,1), a(1,2), a(1,3), \ldots) .
$$

The following notation will be helpful: if $A$ is a numeration system or a counting array, then

$\# n$ is the number of terms of $A$ that are $\leq n$

and do not lie in column 1 of $A$.

LEMMA 3.1. A sequence $S=\left(s_{n}\right)$ is a fractal sequence if and only if the counting array $C(S)$, with terms a $(i, j)$, satisfies

$$
\# a(i, j+1)=a(i, j)
$$

for all $i$ and $j$ in $\mathbb{N}$. 
Proof. It is proved in [3, Theorem 2] that $S$ is a fractal sequence if and only if $C(S)$ is an interspersion. In [3, Lemma 2], it is proved (in different notation) that $C(S)$ is an interspersion if and only if the number of terms of $C(S)$ that lie in column 1 and are not greater than $a(i, j+1)$ is $a(i, j+1)-a(i, j)$. Equivalently, the number of terms of $C(S)$ that lie outside column 1 and are not greater than $a(i, j+1)$ is $a(i, j)$.

Definitions. A finite sequence $S=\left(s_{0}, s_{1}, \ldots, s_{k}\right)$ is a prefractal sequence if the following properties hold:

(PF1) if $i+1=s_{n}$ for some $n \leq k$, then $i=s_{m}$ for some $m<n$, for all $i$ in $\mathbb{N}$;

(PF2) if $\Lambda(S)$ is the sequence obtained from $S$ by deleting the first occurrence of $n$ for each $n$ in $S$, then $\Lambda(S)$ is an initial segment of $S$.

A prefractal basis is a finite basis $\mathbb{B}=\left(b_{0}, b_{1}, \ldots, b_{j}\right)$ such that the first $b_{j}$ terms of $S(\mathbb{B})$ form a prefractal sequence. For example, if $S=$ $(1,1,1,2,1,3,2,1,4,3,2,5,1)$, the first 13 terms in $(5)$, then $\Lambda(S)=$ $(1,1,1,2,1,3,2,1)$, and this is the initial eight-term segment of $S$; thus $S$ is a prefractal sequence, and $(1,2,3,5,8,13)$ is a prefractal basis.

Lemma 3.2. A finite sequence $T=\left(t_{0}, t_{1}, \ldots, t_{k}\right)$ satisfying $(\mathrm{PF} 1)$ is a prefractal sequence if and only if (13) holds for all $i$ and $j$ such that $a(i, j)$ and $a(i, j+1)$ are terms of $C(T)$.

P r o of. The counting array $C(T)$ consists of terms $a(i, j)$ which are the numbers $1, \ldots, t_{k}$. The proof is now similar to that of Lemma 3.1, since all the inequalities needed from [3] and [2] remain intact in the case where the only terms being considered are $1, \ldots, t_{k}$.

LEMmA 3.3. If $1 \leq j_{2}<j_{1}$ and $1 \leq x \leq b_{j_{2}}-1$, then $f_{\mathbb{B}_{j_{1}}}(x)=f_{\mathbb{B}_{j_{2}}}(x)$.

Proof. If $1 \leq x \leq b_{j_{2}}-1$, then the $\mathbb{B}_{j_{1}}$-representation of $x$ and the $\mathbb{B}_{j_{2}}$-representation of $x$ are identical. Thus, the shift-functions defined by (8) have identical values at $x$.

The next theorem shows that the lower bound for $b_{j}$ in Theorem 2(iii) for an affable basis is also a lower bound for $b_{j}$ for a fractal basis. The theorem also gives an upper bound for $b_{j}$.

Theorem 3. Let $\mathbb{B}=\left(b_{0}, b_{1}, \ldots\right)$ be a proper basis, and let $\mathbb{B}_{j}=$ $\left(b_{0}, b_{1}, \ldots, b_{j}\right)$ for $j \geq 2$. The following statements are equivalent:

(i) $\mathbb{B}$ is a fractal basis.

(ii) $\# a(i, j+1)=a(i, j)$ for all $i$ and $j$ in $\mathbb{N}$.

(iii) $f_{\mathbb{B}_{j-1}}\left(b_{j-1}-1\right)+1 \leq b_{j} \leq f_{\mathbb{B}_{j-1}}\left(b_{j-1}\right)$ for all $j \geq 2$.

(iv) $a(i, j+1)=f_{\mathbb{B}}(a(i, j))$ for all $i$ and $j$ in $\mathbb{N}$. 
Proof. A proof is given in four parts: (i) $\Leftrightarrow(i i), \quad(i i i) \Rightarrow(i v)$, (iv) $\Rightarrow(i i)$, and (ii) $\Rightarrow$ (iii).

Part 1: (i) $\Leftrightarrow($ ii). This is an immediate consequence of Lemma 3.2.

Part 2: (iii) $\Rightarrow$ (iv). Suppose, to the contrary, that (iii) holds but (iv) fails. Let $i$ be the least index for which

$$
a(i, j+1) \neq f_{\mathbb{B}}(a(i, j))
$$

for some $j$, and assume that $j$ is the least index such that (14) holds for the stipulated $i$. Write $x$ for $f_{\mathbb{B}}(a(i, j))$. This number must occur somewhere in the array $A(\mathbb{B})$, and then only in column $j+1$ or else row 1 .

Case 1: $x$ in column $j+1$. There is some $h$ for which $a(i, j+1)=$ $f_{\mathbb{B}}(a(h, j))$, and $h>i$, so that $x$ must occur after $f_{\mathbb{B}}(a(h, j))$ in column $j+1$. But now $a(i, j)<a(h, j)$ while $f_{\mathbb{B}}(a(i, j))>f_{\mathbb{B}}(a(h, j))$, contrary to Theorem 2(iv).

Cas e 2: $x$ in row 1 . Here, $x=b_{k}$ for some $k>1$, so that $x=f_{\mathbb{B}}\left(b_{k-1}\right)$. But also, $f_{\mathbb{B}}(a(i, j))=x$, so that $a(i, j)=b_{k-1}$, since, by Theorem $2, f_{\mathbb{B}}$ is strictly increasing. But this implies $i=1$, a contradiction, since we have equality in (14) when $i=1$, by definition of $f_{\mathbb{B}}$.

P art 3: (iv) $\Rightarrow$ (ii). For any $i$ and $j$ in $\mathbb{N}$, let $S_{1}=\{1,2, \ldots, a(i, j)\}$ and $S_{2}=\{m: m \leq a(i, j+1)$ and $m$ is not in column 1 of $A(\mathbb{B})\}$. By (iv), the mapping $f_{\mathbb{B}}$ is a one-to-one correspondence from $S_{1}$ onto $S_{2}$. Therefore, $\# a(i, j+1)=a(i, j)$.

Part 4: (ii) $\Rightarrow$ (iii). Suppose, to the contrary, that (iii) fails. Let $k$ be the least index not less than 2 for which $b_{k}<f_{\mathbb{B}_{k-1}}\left(b_{k-1}-1\right)+1$ or $b_{k}>f_{\mathbb{B}_{k-1}}\left(b_{k-1}\right)$. Let $\widehat{b}_{h}=b_{h}$ for $h=0,1, \ldots, k-1$, and define inductively

$$
\widehat{b}_{k+h}=f_{\widehat{\mathbb{B}}_{k+h-1}}\left(\widehat{b}_{k+h-1}-1\right)+1 \quad \text { and } \quad \widehat{\mathbb{B}}_{k+h}=\left(\widehat{b}_{0}, \widehat{b}_{1}, \ldots, \widehat{b}_{k+h}\right)
$$

for $h=0,1, \ldots$ The basis $\widehat{\mathbb{B}}=\left(\widehat{b}_{0}, \widehat{b}_{1}, \ldots\right)$ satisfies (iii) (with notation modified in an obvious way), so that by Parts 2 and 3 of this proof, already proved, property (ii) holds for the array $A(\widehat{\mathbb{B}})$. That is, $\# \widehat{a}(i, j+1)=\widehat{a}(i, j)$ for all $i$ and $j$ in $\mathbb{N}$, where $\widehat{a}$ denotes terms of $A(\widehat{\mathbb{B}})$.

Case $1: b_{k}<f_{\mathbb{B}_{k-1}}\left(b_{k-1}-1\right)+1$. The number $b_{k-1}-1$ is in $A(\mathbb{B})$, which is to say that it is $a(i, j)$ for some $(i, j)$. The inequality $b_{k-1}-1<b_{k-1}$ can therefore be written as

$$
a(i, j)<a(1, k) .
$$

Now $a(h, j+1)=\widehat{a}(h, j+1)$ for $h=1, \ldots, i-1$, and this accounts for the first $i-1$ terms of column $j+1$ of array $A(\mathbb{B})$. The greatest of these, $a(i-1, j+1)$, is the greatest number that has $\mathbb{B}$-residue less than $b_{k}$. Additionally, the 
number $b_{k-1}+b_{k}=a(1, k)+a(1, k+1)$ is the least number not less than $b_{k}$ whose $\mathbb{B}$-residue is $b_{k-1}$; hence $a(i, j+1)=a(1, k)+a(1, k+1)$, so that

$$
a(i, j+1)>a(1, k+1) .
$$

But since (ii) holds in $A(\mathbb{B})$, the inequalities (15) and (16) are incompatible, and we conclude that $b_{k} \geq f_{\mathbb{B}_{k-1}}\left(b_{k-1}-1\right)+1$.

Case 2: $b_{k}>f_{\mathbb{B}_{k-1}}\left(b_{k-1}\right)$. Let $i$ be the index for which $a(i, 1)=b_{k-1}+$ 1. Then in $A(\widehat{\mathbb{B}})$ we have $f_{\mathbb{B}_{k-1}}(\widehat{a}(i, 1))=f_{\mathbb{B}_{k-1}}\left(b_{k-1}+1\right)$, or equivalently, $\widehat{a}(i, 2)=f_{\mathbb{B}_{k-1}}\left(b_{k-1}\right)+b_{1}$. Now $a(h, 2)=\widehat{a}(h, 2)$ for $h=1, \ldots, i-1$, and this accounts for the first $i-1$ terms of column 2 of $A(\widehat{\mathbb{B}})$. Since $\widehat{a}(i, 2)$ and $b_{1}$ both have $\mathbb{B}$-residue $b_{1}$, their difference, $f_{\mathbb{B}_{k-1}}\left(b_{k-1}\right)$, also has $\mathbb{B}$-residue $b_{1}$, so that

$$
a(i, 2) \leq f_{\mathbb{B}_{k-1}}\left(b_{k-1}\right)=a(1, k+1) .
$$

We now have $a(i, 1)>a(1, k)$ and $a(i, 2) \leq a(1, k+1)$, contrary to (ii). Therefore, $b_{k} \leq f_{\mathbb{B}_{k-1}}\left(b_{k-1}\right)$.

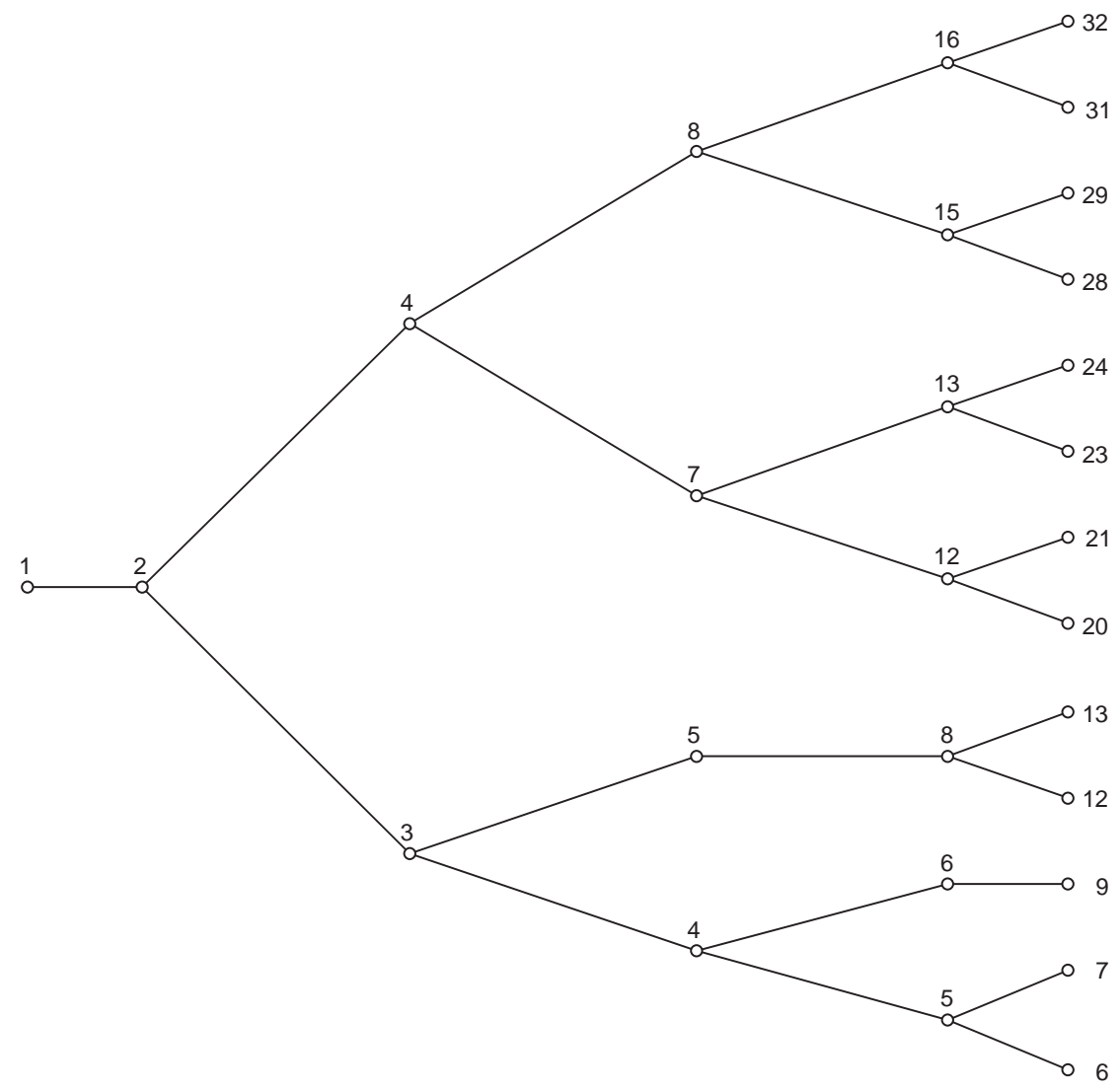

Fig. 1. The first six terms of the fractal bases in which $b_{1}=2$ 
Corollary 3.1. With reference to statement (iii) in Theorem 3, the number of allowable $b_{j}$ is not greater than $b_{1}$.

Proof. By Theorem 3, the greatest allowable $b_{j}$ is the number $f_{\mathbb{B}_{j-1}}\left(b_{j-1}\right)$, which we abbreviate as $M$. In the array $A(\mathbb{B})$, the consecutive integers

$$
M-1, M-2, \ldots, M-\left[f_{\mathbb{B}_{j-1}}\left(b_{j-1}\right)-f_{\mathbb{B}_{j-1}}\left(b_{j-1}-1\right)-1\right]
$$

all lie in column 1 , since none of them is of the form $f_{\mathbb{B}_{j-1}}(x)$. It follows easily from the definition of $\mathbb{B}_{j-1}$-representation and Theorem $3(\mathrm{iii})$, that the maximum number of consecutive integers for which this is possible is $b_{1}$.

Corollary 3.2. Let $\mathbb{B}=\left(b_{0}, b_{1}, \ldots\right)$ be a proper basis. Suppose $\mathbb{B}_{j_{1}}=$ $\left(b_{0}, b_{1}, \ldots, b_{j_{1}}\right)$ is a prefractal basis for all $j_{1} \geq 1$, and $b_{j}=f_{\mathbb{B}_{j-1}}\left(b_{j-1}\right)$ for $j=j_{1}+1, j_{1}+2, \ldots$ Then $\mathbb{B}$ is a fractal basis. If the $\mathbb{B}_{j_{1}-1}$-representation of $b_{j_{1}}$ is given by

$$
b_{j_{1}}=\gamma_{j_{1}-p} b_{j_{1}-p}+\gamma_{j_{1}-p+1} b_{j_{1}-p+1}+\ldots+\gamma_{j_{1}-1} b_{j_{1}-1}
$$

then the row sequences of $A(\mathbb{B})$ satisfy the homogeneous linear recurrence inherited from (17):

$a\left(i, j_{1}+q\right)$ $=\gamma_{j_{1}-p} a\left(i, j_{1}+q-p\right)+\gamma_{1} a\left(i, j_{1}+q-p+1\right)+\ldots+\gamma_{j_{1}-1} a\left(i, j_{1}+q-1\right)$, $i \geq 1, q \geq 0$.

Proof. This is an obvious consequence of Theorem 3(iv).

In summary, a prefractal basis $\left(b_{0}, b_{1}, \ldots, b_{j}\right)$ can always be extended to a prefractal basis $\left(b_{0}, b_{1}, \ldots, b_{j}, b_{j+1}\right)$, where the number $p$ of allowable values of $b_{j+1}$ satisfies $1 \leq p \leq b_{1}$. Extending inductively, we can in this manner construct any fractal basis as a limit of prefractal bases. If the choice of $b_{j+1}$ is always maximal beginning with the first term after some particular $b_{j_{1}}$, then we obtain, in accord with Corollary 3.2, the homogeneous extension of $\left(b_{0}, b_{1}, \ldots, b_{j_{1}}\right)$. Specifically, if $b_{j_{1}}$ is given by the $\mathbb{B}_{j_{1}-1}$-representation

$$
b_{j_{1}}=\mathcal{R}\left(b_{0}, b_{1}, \ldots, b_{j_{1}-1}\right)=\gamma_{0} b_{0}+\gamma_{1} b_{1}+\ldots+\gamma_{j_{1}-1} b_{j_{1}-1},
$$

then all the row sequences of the limiting fractal basis satisfy the homogeneous recurrence determined by $\mathcal{R}$. We now turn to certain nonhomogeneous linear recurrences, associated with minimal choices of $b_{j+1}$, as given by (20).

Corollary 3.3. Suppose $\mathbb{B}_{j_{1}}=\left(b_{0}, b_{1}, \ldots, b_{j_{1}}\right)$ is a prefractal basis for all $j_{1} \geq 1$. Let the $\mathbb{B}_{j_{1}}$-representation of $b_{j_{1}}-1$ be given by

$$
b_{j_{1}}-1=\delta_{j_{1}-p} b_{j_{1}-p}+\delta_{j_{1}-p+1} b_{j_{1}-p+1}+\ldots+\delta_{j_{1}-1} b_{j_{1}-1},
$$


where $\delta_{j_{1}-p} \neq 0$, so that the order of the homogeneous linear recurrence $\mathcal{S}$ given by

$$
\mathcal{S}\left(x_{1}, x_{2}, \ldots, x_{p}\right)=\delta_{j_{1}-p} x_{1}+\delta_{j_{1}-p+1} x_{2}+\ldots+\delta_{j_{1}-1} x_{p}
$$

is $p$. Let $\mathbb{B}$ be the fractal basis obtained inductively from $\mathbb{B}_{j_{1}}$ by defining

$$
b_{j_{1}+q+1}=f_{\mathbb{B}_{j_{1}+q}}\left(b_{j_{1}+q}-1\right)+1
$$

for $q=0,1, \ldots$ Then row 1 of $A(\mathbb{B})$ satisfies the nonhomogeneous linear recurrence

$$
\begin{aligned}
b_{j} & =a(1, j+1)=\mathcal{S}\left(b_{j-p}, b_{j-p+1}, \ldots, b_{j-1}\right)+1 \\
& =\mathcal{S}(a(1, j-p+1), a(1, j-p+2), \ldots, a(1, j))+1,
\end{aligned}
$$

for $j=j_{1}, j_{1}+1, \ldots$, and row $i$ of $A(\mathbb{B})$ satisfies the nonhomogeneous linear recurrence

$$
a(i, j+1)=\mathcal{S}(a(i, j-p+1), a(i, j-p+2), \ldots, a(i, j))+Q_{i},
$$

where $Q_{i}$ depends only on $i$, for all $i$ in $\mathbb{N}$, for $j=j_{1}, j_{1}+1, \ldots$

P r o of. Equations (18) and (19) give

$$
f_{\mathbb{B}_{j_{1}}}\left(b_{j_{1}}-1\right)=f_{\mathbb{B}_{j_{1}}}\left(\mathcal{S}\left(b_{j_{1}-p}, b_{j_{1}-p+1}, \ldots, b_{j_{1}-1}\right)\right),
$$

so that by (20) with $q=0$, we have $b_{j_{1}+1}=\mathcal{S}\left(b_{j_{1}-p+1}, b_{j_{1}-p+2}, \ldots, b_{j_{1}}\right)+1$. The same method easily completes an induction proof that (21) holds for all $j \geq j_{1}$, so that (22) is established for $i=1$.

Assume now that $i \geq 2$ and $j \geq j_{1}$. Let the $\mathbb{B}$-representation of $a(i, 1)$ be given by $a(i, 1)=\sum_{h=1}^{v} c_{h-1} a(1, h)$, and let $Q_{i}=\sum_{h=0}^{v-1} c_{h}$. By Theorem 3 ,

$$
\begin{aligned}
a(i, j+1) & =\sum_{h=1}^{v} c_{h-1} a(1, j+h) \\
& =\sum_{h=1}^{v} c_{h-1}\left(\mathcal{S}\left(b_{j-p+h-1}, b_{j-p+h}, \ldots, b_{j+h-1}\right)+1\right) \\
& =Q_{i}+\sum_{h=1}^{v} c_{h-1} \sum_{k=0}^{p-1} \delta_{j-p+k} b_{j-p+k} \\
& =Q_{i}+\sum_{k=1}^{p} \delta_{j-k} \sum_{h=1}^{v} c_{h-1} a(1, j-k+h) \\
& =Q_{i}+\sum_{k=1}^{p} \delta_{j-k} a(i, j-k+1) \\
& =\mathcal{S}(a(i, j-p+1), a(i, j-p+2), \ldots, a(i, j))+Q_{i} .
\end{aligned}
$$




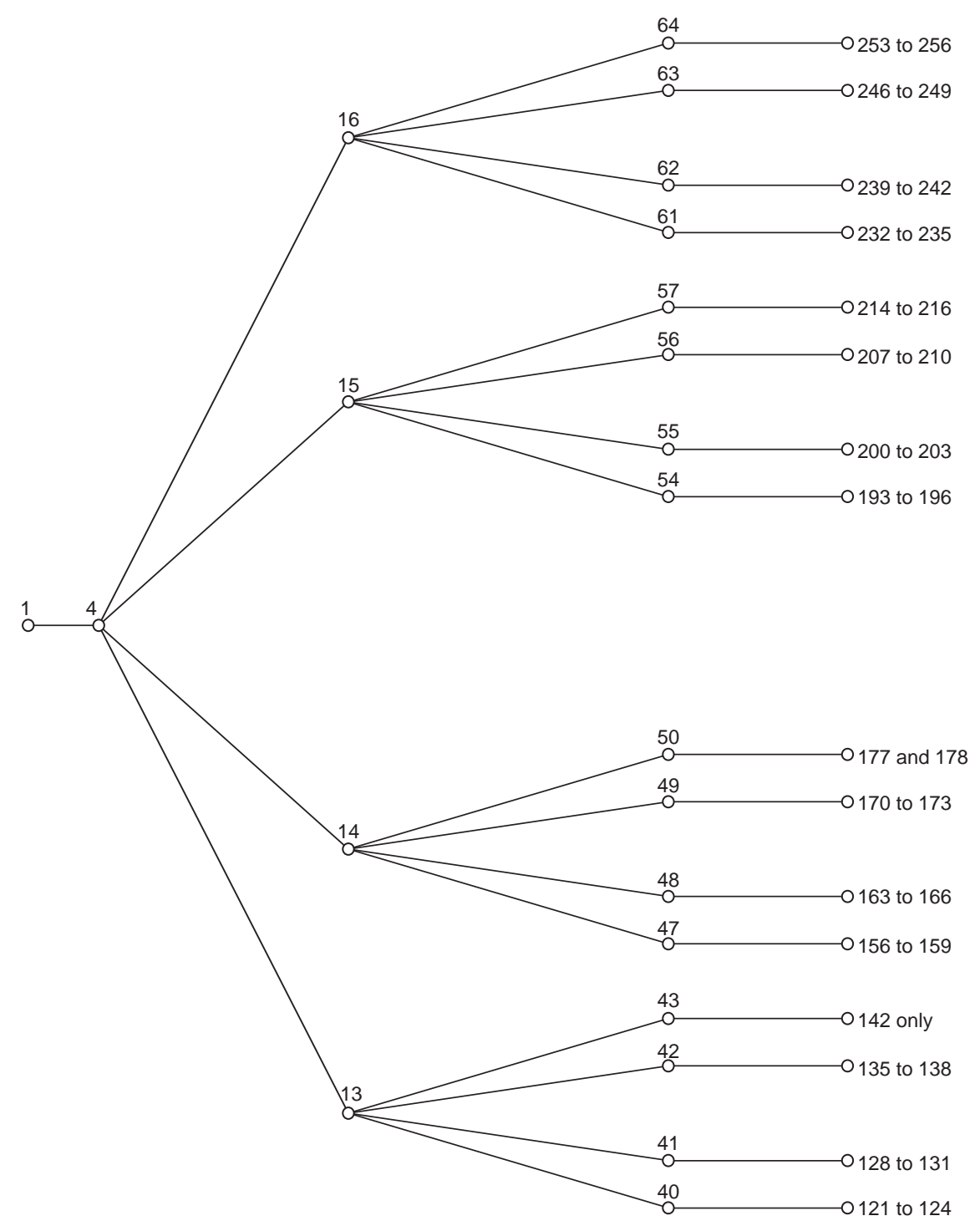

Fig. 2. The first five terms of the fractal bases in which $b_{1}=4$

Figures 1 and 2 lead one to conjecture that every prefractal basis has uncountably many extensions to fractal bases. Another question concerns the inequality in Theorem 3(iii): when is there only one possible choice of $b_{j}$, as exemplified by $b_{4}=8$ following $b_{3}=5$ in Figure 1 , and also by $b_{4}=142$ following $b_{3}=43$ in Figure 2?

Finally, as you may have already observed, for each choice of $b_{1} \geq 2$, the fractal bases with second term $b_{1}$ fan themselves out between two extreme cases, one an arithmetic sequence and the other a geometric sequence. 


\section{References}

[1] A. Fraenkel, Systems of numeration, Amer. Math. Monthly 92 (1985), 105-114.

[2] C. Kimberling, Interspersions and dispersions, Proc. Amer. Math. Soc. 117 (1993), 313-321.

[3] —, Fractal sequences and interspersions, Ars Combin., to appear.

[4] A. M. Yaglom and I. M. Yaglom, Challenging Mathematical Problems With Elementary Solutions, Vol. II, Dover, New York, 1967.

DEPARTMENT OF MATHEMATICS

UNIVERSITY OF EVANSVILLE

EVANSVILLE, INDIANA 47722

U.S.A.

Received on 17.6.1994

and in revised form on 12.1.1995 